\title{
The Optimized Cell Configuration METHOD OF AVOIDING SRS INTER-CELL INTERFERENCE
}

\author{
Shin-Hwan Kim ${ }^{1}$, Kyung-Yup Kim ${ }^{1}$, Jae-Hyung Koo ${ }^{2}$ \\ and Young-Soo $\mathrm{Seo}^{3}$ \\ ${ }^{1}$ Access Network Technology Team, Korea Telecom, Seoul, Korea \\ ${ }^{2}$ Access Network Technology Department, Korea Telecom, Seoul, Korea \\ ${ }^{3}$ Network Research Technology Unit, Korea Telecom, Seoul, Korea
}

\begin{abstract}
The issue of cell-to-cell interferences is a serious problem that has always been raised in digital communication system such as NR. The communication method of NR and LTE is OFDM. OFDM has many advantages, but has fatal disadvantage called ICI (Inter-Cell Interference) because resources among cells are always overlapped. For example, NR's typical interferences are ICIs among PDSCH (Physical Downlink Shared Channel), PDCCH (Physical Downlink Control Channel), PUSCH (Physical Uplink Shared Channel), PUCCH (Physical Uplink Control Channel), CSI-RS (Channel State Information-Reference Signal) and SRS (Sounding Reference Signal). Among them, it is important to determine the correct beamforming weight factor value by estimating the channel with SRS. Therefore, the ICI of SRS degrades the performance of downlink throughput. This paper analyses the impact of SRS'S ICI in conventional scheme, introduces the proposed AC-CS (Auto-Correlation Cyclic Shift) schemes by the Zadoff-Chu sequence to overcome the ICI of SRS and analyses theirs performance. The method used for performance analysis is determined by the detection abilities, which are missing probability and false alarm probability.
\end{abstract}

\section{KEYWORDS}

SRS, beamforming, auto-correlation, missing probability, false alarm probability

\section{INTRODUCTION}

In digital communication system such as LTE and NR, the inter-cell interference issue is a problem that has always been raised particularly. In order to solve this interference problem in the OFDM communication method, but the like algorithms such as ICIC (Inter-Cell Interference Cancellation) or CoMP (Coordinated Multi-Point) are emerged as a solution, there are disadvantages such as dividing the resources used.

In particular, the estimated performance by the received SRS (Sounding Reference Signal) distortion in the serving cell due to the SRS of the adjacent cells to derive a weight factor of the inaccurate beamforming is significantly reduced in condition of the low power or interference.

Thus, first of all, this paper analyses the performance degradation of estimated SRS due to adjacent cell's SRS in the conventional conditions that are currently commercialized 5G system, compares the simulation results in the proposed AC-CS (Auto-Correlation Cyclic Shift) schemes

David C. Wyld et al. (Eds): SOFEA, CTCM, BIBC, SIPR, NCWC, CSEN, EDTECH - 2021

pp. 107-116, 2021. CS \& IT - CSCP 2021

DOI: $10.5121 /$ csit.2021.111609 
of the Zadoff-Chu sequence and the conventional scheme in the cell's configuration and recommends the conditionally-fitted schemes of each a morphology.

\section{BACKGROUND}

\subsection{Sounding Reference Signal}

SRS is the acronym of Sounding Reference Signal. When the UE transmits to gNB to estimate the status of the uplink channel sent to $\mathrm{gNB}$, gNB uses a way to report to the UE based on the channel status it currently identifies and reflect on future UL. In particular, it is importantly used in MIMO (Multi-Input Multi-Output), which is referred to as the leading technology in NR.

First of all, the UE can transmit the SRS to beam in several directions. gNB understands the channel status in each direction based on SRS of receiver, which gives the uplink channel the information it needs, such as which beam to use and how much rank to use. Based on the reporting result of the received $\mathrm{gNB}$, the UE proceeds uplink process. Here's one thing to go through about DL/UL's CSI (Channel State Information).

If UL and DL in FDD use different frequencies, each sub-band CSI is different. However, in UL and DL in TDD using the same frequency band divided only in time, there is a point to consider. Since the factors that determine the channel state are largely composed of interference and obstacles of the frequency, there is a concept called channel reciprocity in TDD.

\subsection{Zadoff-Chu Sequence}

Zadoff-Chu sequences exhibit the useful property that cyclically shifted versions of themselves are orthogonal to one another, provided that each cyclic shift, when viewed within the time domain of the signal, is greater than the combined propagation delay and multipath delay spread of the signal between the transmitter and receiver.

A generated Zadoff-Chu sequence that has not been shifted is known as a root sequence. The complex value at each position $n$ of each root Zadoff-Chu sequence parametrized by $u$ is given by

$$
x_{u}(n)=e^{-j\left(\frac{\pi u n(n+1)}{N_{Z C}}\right)}
$$

Where $0 \leq \mathrm{n}<\mathrm{N}_{\mathrm{ZC}}, 0<u<\mathrm{N}_{\mathrm{ZC}}, \operatorname{gcd}\left(\mathrm{N}_{\mathrm{ZC}}, \mathrm{u}\right)=1$ and $\mathrm{N}_{\mathrm{ZC}}=$ length of sequence.

\subsection{Properties of Zadoff-Chu sequence}

- They are periodic with period $\mathrm{N}_{\mathrm{ZC}}$ if $\mathrm{N}_{\mathrm{ZC}}$ is odd.

- If $\mathrm{N}_{\mathrm{ZC}}$ is prime, the DFT (Discrete Fourier Transform) of a Zadoff-Chu sequence is another Zadoff-Chu sequence conjugated, scaled and time scaled.

- The auto correlation of a Zadoff-Chu sequence with a cyclically shifted version of itself is zero, i.e., it is non-zero only at one instant which corresponds to the cyclic shift.

- The cross-correlation between two prime length Zadoff-Chu sequences, i.e. different values of $u, u=u_{1}, u=u_{2}$, is constant $1 / \sqrt{N_{Z C}}$, provided that $u_{1}-u_{2}$ is relatively prime to $\mathrm{N}_{\mathrm{ZC}}$. 


\subsection{Auto-correlation}

Auto-correlation is an indicator of the similarity between source sequence and sequence of added noise channel. Also, it is a time series and its simple linear correlation with its own past.

Basically, the value $x(t)$ means the correlation with the sequence of the sequence and the value $\mathrm{x}(\mathrm{t}+\theta)$ after the $\theta$ in hours. The time displacement $\theta$ is called time difference. Auto-correlation function is a correlation related to variable time. The coefficient of auto-correlation relates to the variable $\mathrm{x}(\mathrm{t})$ and the variable $\mathrm{x}(\mathrm{t}+\theta)$ as a product-moment correlation coefficient.

\subsection{Cross-correlation}

In digital signal processing, cross-correlation is a level of similarity of two different sequences as a function of one another relationship. Simply, it is a correlation between two sequences $x(t)$ and $y(t)$.

Two sequences are the same variable measured in different places or the same variable observed in the same place, which may have different observation times. As an example, $y(t)$ may represent $\mathrm{x}(\mathrm{t}+\theta)$, wherein $\theta$ means a time delay.

\section{SRS SEQUENCE GROUP IN NR}

For example, the sequence group $\mathrm{u}=\left(\mathrm{n}_{\mathrm{ID}}^{\mathrm{SRS}}\right) \bmod 30$ if groupOrSequenceHopping equals 'neither', neither group, nor sequence hopping shall be used. The SRS sequence identityn $\mathrm{n}_{\mathrm{ID}}^{\mathrm{SR}}$ is given by the higher layer parameter sequenceId in the SRS-Config IE, in which case $\mathrm{n}_{\mathrm{ID}}^{\mathrm{SRS}} \in$ $\{0,1, \ldots, 1023\}$ and is PCID (Physical Cell Identifier) generally.

\section{Conventional Cell Configuration by SRS}

The 5G commercialized vendors around the world use $\mathrm{n}_{\mathrm{ID}}^{\mathrm{SRS}}$ to PCID (Physical Cell Identifier) generally.

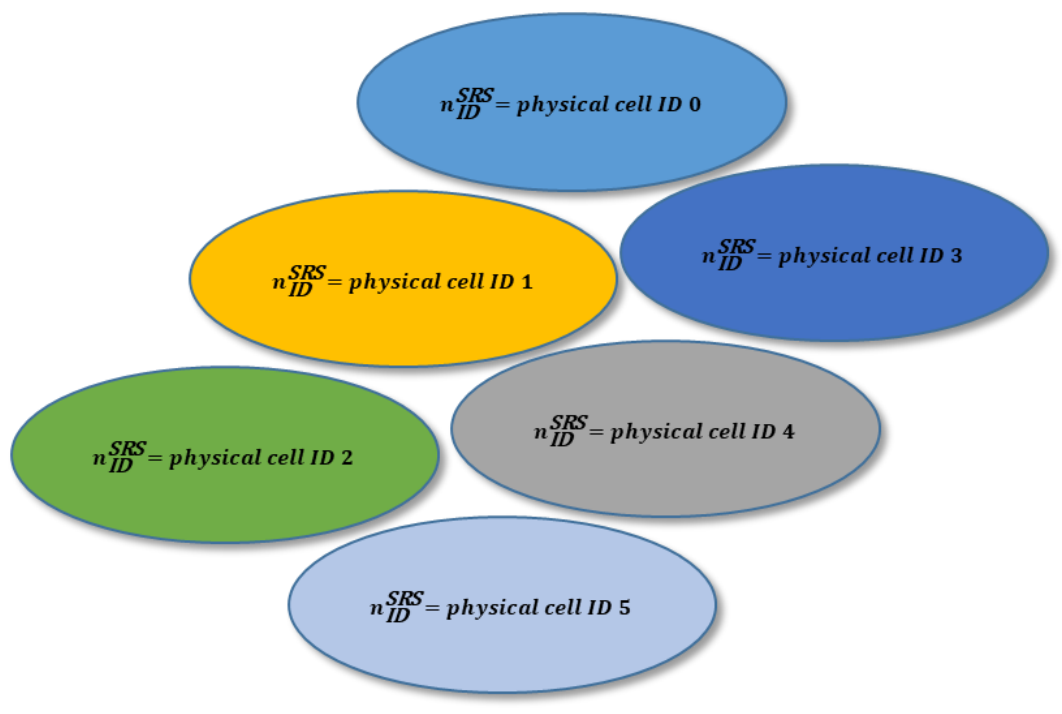

Figure 1. Conventional sequence group ID by SRS 
In this case, the group ID is used differently between cells, so the type of Zadoff-Chu sequence is different between cells, as expressed in different colours, such as Figure 1. When decoding the received signal from the position of the base station, the signal coming from the other cell is to act as interference. In an environment such as Figure 1, because the SRS group id is different for each cell, it has the characteristics of cross-correlation rather than the characteristics of autocorrelation.

Since Zadoff-Chu sequence of other cell using other SRS group ID increases noise by $\frac{1}{\sqrt{\mathrm{N}_{\mathrm{ZC}}}}$ as mentioned above, the performance can only be degraded in environments such as UMa (Urban Macro) with many surrounding cells. Vendors are to configure the settings differently according to morphology, such as RMa (Rural Macro) or UMa (Urban Macro), so it has a very complex configuration, from a commercialization point of view.

All currently commercialized vendors want to be a stable and simple configuration. Therefore, they choose to configure the SRS group ID with PCID (Physical Cell ID). However, this method will reduce performance in the cell edge in an environment such as UMa (Urban Macro). Performance decreases with the number of nested cells is in the simulation results in the performance section below.

\section{Proposed Cell Configuration Schemes}

In order to overcome the degradation of conventional scheme such as Figure 1, two new schemes are proposed. Conventional method, the more nested cells, is the more disadvantageous of increasing the noise by the cross-correlation characteristics.

The two newly proposed methods are using the same SRS sequence to reduce the noise due to cross-correlation of the surrounding cells to overcome the conventional disadvantages, and between cells using the same sequence is applied a method of minimizing interference using a cyclic shift.

\section{1. $1^{\text {st }}$ Proposed Scheme}

In the cell configuration, as shown in the example of Figure $2,1^{\text {st }}$ proposed scheme is a method of using the same SRS group ID in three cells' unit. That is, this scheme uses the same SRS sequence in three cells' unit, and they use a same sequence applied to different cyclic shift. At this time, other three cells can be deployed by same configuration. Blue cells use the same SRS sequence having the same SRS group ID, and that sequence is done by different cyclic shift. Green cells also use the same configuration.

The NR RRC message used can be configured as Table 1 with the PCID (Physical Cell Identify) of each cell, which is constituted by the cyclic shift with modulo 3. At this time, the shifted sequence should be configured as far away as possible for a good performance. For example, it is best as far as 4 units of 12 intervals to maximize performance in this condition of NR. The reason is that the peak by auto-correlation of the same sequence is not ideal and the peak is gradually growing. This shows in the simulation results' section below. The combOffset- $n 4$ and cyclicShift$n 4$ are RRC Messages for the distinction of UEs.

If the cell is configured as the same SRS Group ID of three cells' unit, when there are many adjacent cells, such as Figure 2, there is still disadvantage that noise is increased by the interference of cross-correlation from cells of different colour. 
The way to overcome this disadvantage is a $2^{\text {nd }}$ proposed scheme.

Table $1.1^{\text {st }}$ proposed cell configuration

\begin{tabular}{|c|c|c|c|}
\hline SRS RRC Message & Usage & \multicolumn{2}{|c|}{ Value } \\
\hline combOffset- $n$ 4 & Intra-Cell UEs & \multicolumn{2}{|c|}{$0,1,2,3$} \\
\hline \multirow{3}{*}{ cyclicShift- $n 4$} & \multirow{2}{*}{ Inter-Cell UEs } & PCID \% 3=0 & 0 \\
\cline { 3 - 4 } & & PCID \% 3=1 & 4 \\
\cline { 3 - 4 } & & PCID \% 3 =2 & 8 \\
\hline
\end{tabular}

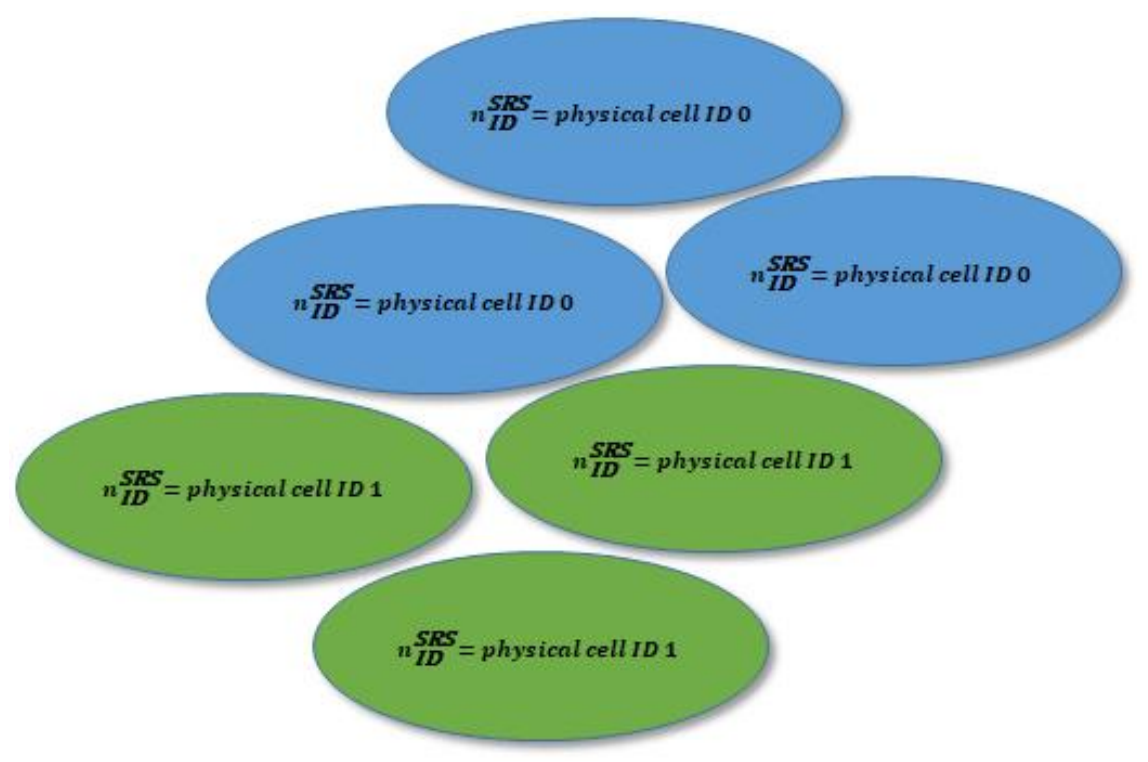

Figure 2. $1^{\text {st }}$ proposed cell configuration by SRS sequence group ID

\section{2. $2^{\text {nd }}$ Proposed Scheme}

As in the example of Figure 3, the $2^{\text {nd }}$ proposed scheme is a method of using the same SRS group ID in six cell's unit in the cell configuration. That is, this scheme uses the same SRS sequence in six cells' unit, and they use a same sequence applied to different cyclic shift. At this time, cells of different colour can be deployed by same configuration. That is, blue cells are the same SRS sequence using the same SRS group ID and the sequence is done by different cyclic shift.

Table 2. $2^{\text {nd }}$ proposed cell configuration

\begin{tabular}{|c|c|c|c|}
\hline SRS RRC Message & Usage & \multicolumn{2}{|l|}{ Value } \\
\hline combOffset-n4 & Intra-Cell UEs & \multicolumn{2}{|l|}{$0,1,2,3$} \\
\hline \multirow{6}{*}{ cyclicShift-n4 } & \multirow{6}{*}{ Inter-Cell UEs } & PCID $\% 6=0$ & 0 \\
\hline & & PCID \% 6 = 1 & 2 \\
\hline & & PCID $\% 6=2$ & 4 \\
\hline & & PCID $\% 6=3$ & 6 \\
\hline & & PCID \% $6=4$ & 8 \\
\hline & & PCID \% $6=5$ & 10 \\
\hline
\end{tabular}




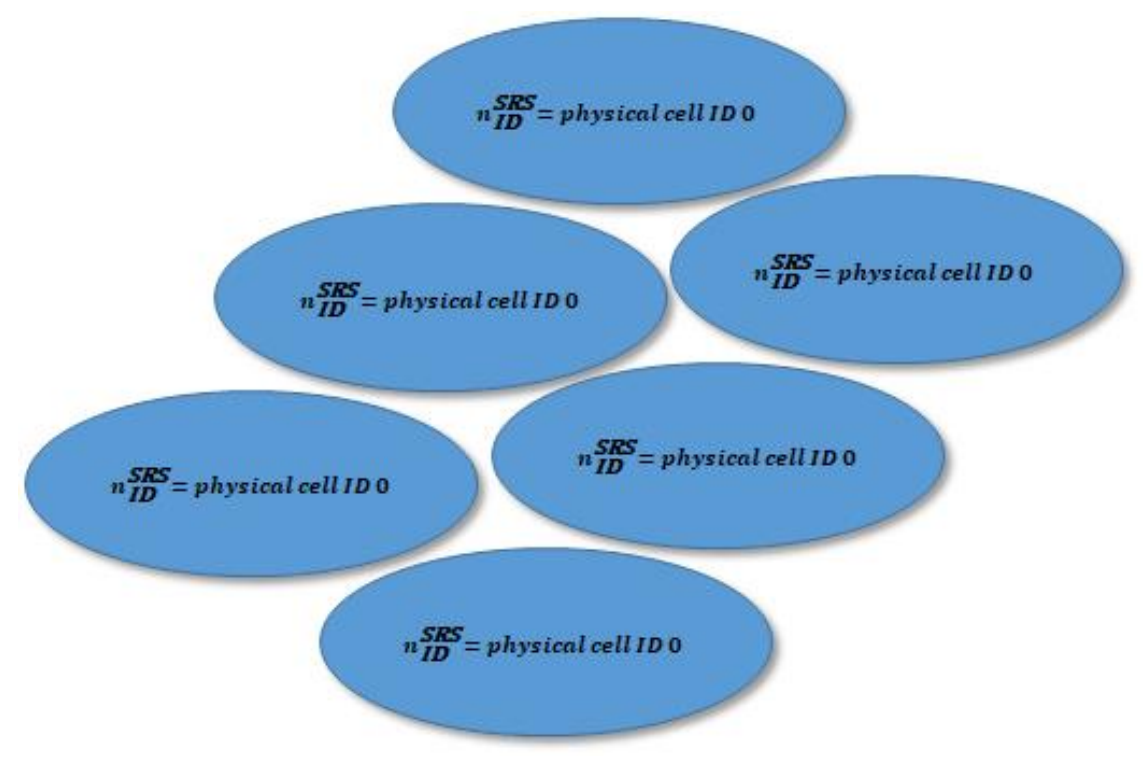

Figure 3. $2^{\text {nd }}$ proposed cell configuration by SRS sequence group ID

The NR RRC message used can be configured as Table 2 with the PCID (Physical Cell Identify) of each cell, which is constituted by the cyclic shift with modulo 6. At this time, the shifted sequence should be configured as far away as possible for good performance. For example, it is best as far as 2 units of 12 intervals to maximize performance in this condition of NR. The reason is that peak by auto-correlation of the same sequence is not ideal and the peak is gradually growing. This also shows in the simulation results' section below.

\subsection{Pros and Cons}

The pros and cons for each scheme are expressed as table 3. Conventional scheme has an advantage that the cell configuration is simple, but there is a disadvantage that the SRS crosscorrelation interference between cells is increased. To compensate for this disadvantage, the $1^{\text {st }}$ proposed scheme has the best auto-correlation characteristics, but since only three cells are the same sequence, there is some SRS cell-to-cell interference. The $2^{\text {nd }}$ proposed scheme is that configured all the cells ( 6 cells) have the same sequence. This method has an advantage that SRS inter-cell interference does not exist and the disadvantage that the characteristics of autocorrelation of closerpeakby cyclic shift in same sequence may be worse.

Table 3. Each Scheme Comparison

\begin{tabular}{|c|c|c|}
\hline \multirow{2}{*}{ Scheme } & \multicolumn{2}{|c|}{ Comparison } \\
\cline { 2 - 3 } & Advantage & Disadvantage \\
\hline Conventional & Easy cell configuration & High SRS ICI $^{\mathrm{a}}$ \\
\hline $1^{\text {st }}$ Proposed & Good auto-correlation $^{\text {ny }}$ & Medium SRS ICI $^{\mathrm{a}}$ \\
\hline $2^{\text {nd }}$ Proposed & Low SRS ICI $^{\mathrm{a}}$ & Medium auto-correlation \\
\hline
\end{tabular}




\section{Performance Comparison}

Based on what we've described so far, compare the performance of the conventional, $1^{\text {st }}$, and $2^{\text {nd }}$ proposed scheme.

First of all, Figure 4 is the channel correlation of $1^{\text {st }}$ proposed scheme and Figure 5 is the channel correlation of $2^{\text {nd }}$ proposed scheme. According to Figure 4 and Figure 5, the $1^{\text {st }}$ proposed scheme is cleaner around peak than the $2^{\text {nd }}$ proposed scheme. The reason is that the $1^{\text {st }}$ proposed scheme has low number of cyclic shift, so the overall noise can be elevated, but false alarm probability is reduced than $2^{\text {nd }}$ proposed scheme such as Figure 7.

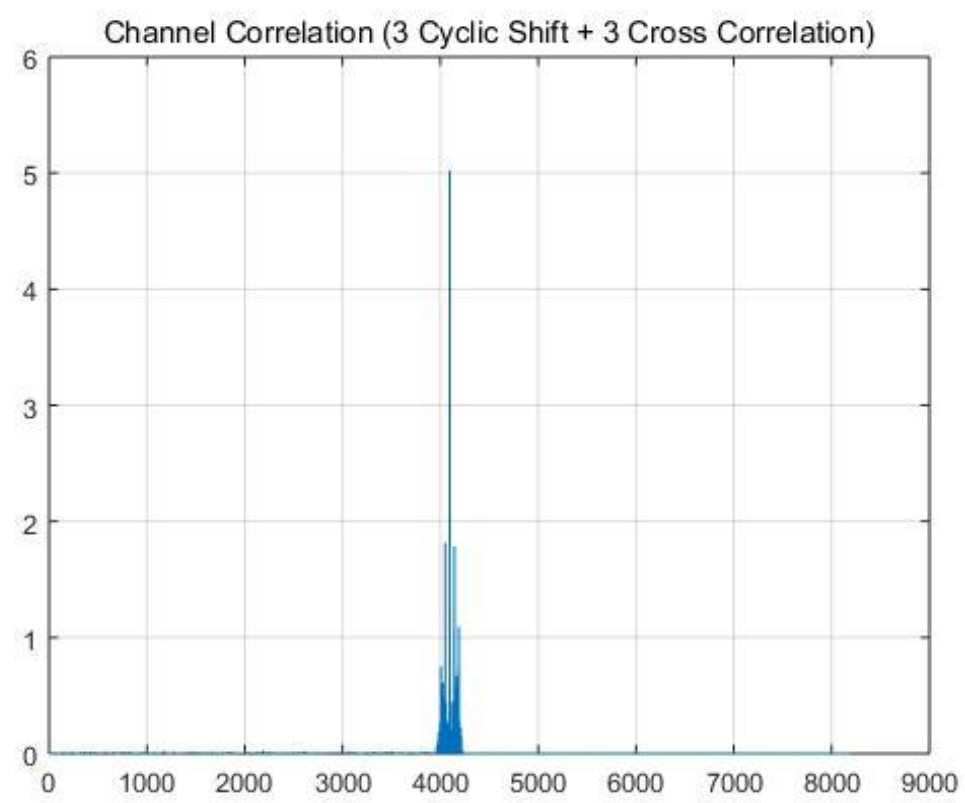

Figure 4. The channel correlation property of $1^{\text {st }}$ proposed scheme

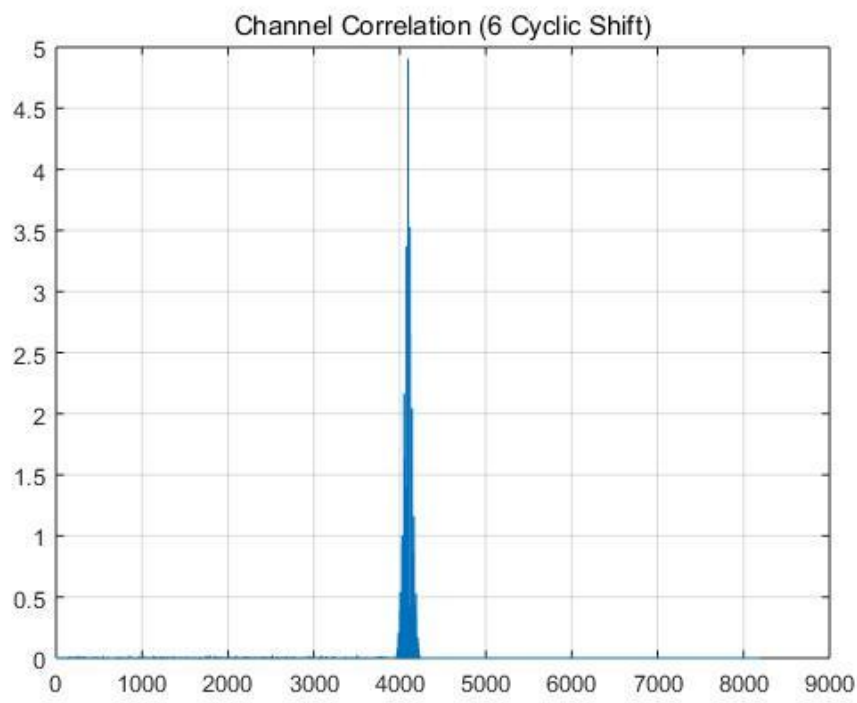

Figure 5. The channel correlation property of $2^{\text {nd }}$ proposed scheme 


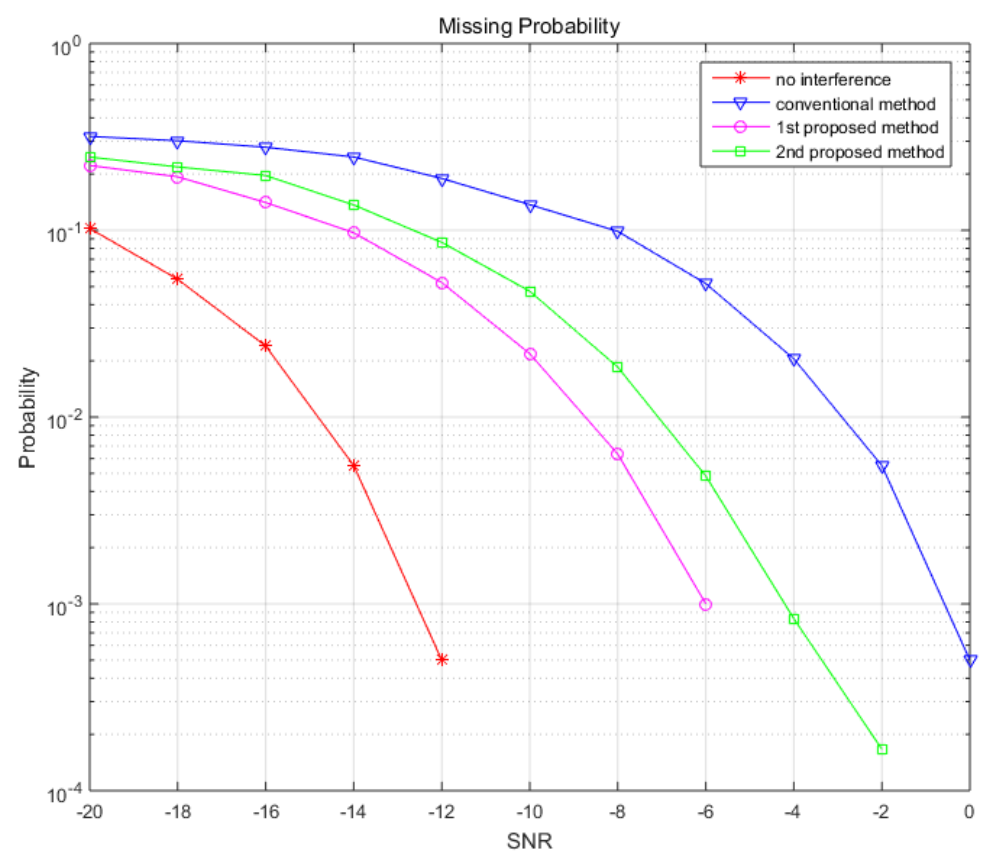

Figure 6. The Performance of no ICI, conventional and proposed scheme

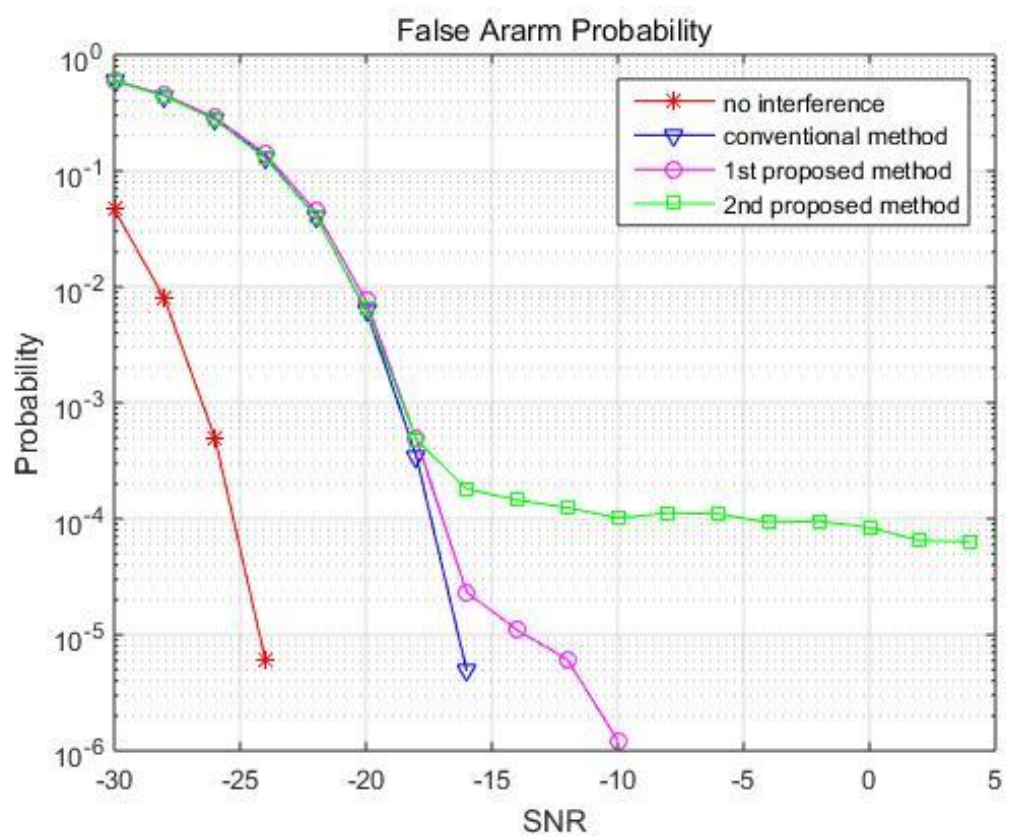

Figure 7. The False Alarm of no ICI, conventional and proposed scheme

The graph of comprehensive performance is Figure 6. Based on $1 \%$ missing probability, the conventional scheme has bad performance by $12 \mathrm{~dB}$ compared to no interference. However, $1^{\text {st }}$ proposed scheme has good $6 \mathrm{~dB}$ performance compared to a conventional scheme, and $2^{\text {nd }}$ proposed scheme has $4 \mathrm{~dB}$ gain compared to a conventional scheme.

Figure 7 is the comparison of false alarm probability in same condition of Figure 6 . The $2^{\text {nd }}$ proposed scheme looks relatively poor in good SINR conditions. The reason is that there are a lot of small peaks in addition to real peak due to the cyclic shift of same SRS sequence. However, 
there is no issue of performance degradation since 3GPP standard recommends the false alarm conditions satisfy $0.1 \%$ generally.

Based on the performance graph, the morphology which $1^{\text {st }}$ scheme is used in commercial environment is appropriate urban regions in typical, and $2^{\text {nd }}$ scheme is a more suitable method in dense urban region where cells are tighter. Because, the tighter the cell, the worse the crosscorrelation characteristics, so dense urban region is good to use the method of cyclic shift as much as possible with the same SRS sequence.

\section{Conclusions}

It is very important in digital beamforming algorithm to determine the correct beamforming weight factor by estimating the channel with uplink SRS. Thus, this paper analysed the level of interference between the cells receiving the SRS in various ways.

Using the new proposed AC-CS (Auto-Correlation Cyclic Shift) of Zadoff-Chu sequence, base station can overcome inter-cell interference when it receives SRS. Also, we compared its performance with conventional and proposed schemes. In order to maximize performance in the actual commercial environment, $1^{\text {st }}$ and $2^{\text {nd }}$ proposed schemes can be selected depending on the specific morphology.

\section{ACKNOWLEDGEMENTS}

The authors would like to thank everyone and coordination of company especially.

\section{REFERENCES}

[1] D. V. Sarwate, "Bounds on correlation and autocorrelation of sequences," IEEE Trans. Inf. Theory, vol. IT-25, no. 6, pp. 720-724, Nov. 1979.

[2] M. B. Çelebi and H. Arslan, "Theoretical analysis of the co-existence of LTE-A signals and design of an ML-SIC receiver," IEEE Trans. Wirel.Commun., vol. 14, no. 8, pp. 4626-4639, Aug. 2015.

[3] J. W. Kang, Y. Whang, H. Y. Lee, and K. S. Kim, "Optimal pilot sequence design for multi-cell MIMO-OFDM systems," IEEE Trans. Wireless Commun., vol. 10, no. 10, pp. 3354-3367, Oct. 2011.

[4] T. Kasami, Weight Distribution Formula for Some Class of Cyclic Codes, Coordinated Science Lab., Univ. Illinois Urbana-Champaign, 1966, Tech. Rep. R285.

[5] R. Gold, "Maximal recursive sequences with 3-valued recursive crosscorrelation functions," IEEE Trans. Inf. Theory, vol. 14, no. 1, pp. 154-156, Jan. 1968.

[6] S. Bozta, R. Hammons, and P. V. Kummer, "4-phase sequences with near optimum correlation properties," IEEE Trans. Inf. Theory, vol. 38, no. 3, pp. 1101-1113, May 1992.

[7] P. Fan, M. Darnell, and F. Fan, Sequence Design for Communications Applications, 1st ed. New York: Taylor \& Francis, 1996.

[8] D. V. Sarwate and M. B. Pursley, "Crosscorrelation properties of pseudorandom and related sequences,” Proc. IEEE, vol. 68, no. 5, pp. 593-619, May 1980.

[9] B. M. Popovic, "Generalized chirp-like polyphase sequences with optimum correlation properties," IEEE Trans. Inf. Theory, vol. 38, no. 4, pp. 1406-1409, Jul. 1992.

[10] L. R. Welch, "Lower bounds on the maximum cross correlation of signals," IEEE Trans. Inf. Theory, vol. 20, no. 3, pp. 397-399, May 1974.

[11] B B. Ramakrishnan, Matched nonlinearities for frame synchronization in presence of frequency offsets[J], Electronics Letters, 2009, Vol.45, No.4, pp.236-237.

[12] R. Pedone, M. Villani, A. Vanelli-Coralli, et al., Frame Synchronization in Frequency Uncertainty[J], IEEE Transactions on Communications, 2010, Vol. 58, No. 4, pp. 1235-1246.

[13] A. Korpansky and M. Bystrom, Detection of aperiodically embedded synchronization patterns[J], IEEE Transactions on Communications, 2004, vol. 3, no. 5, pp. 1386-1392. 
[14] Matsumoto, Y., Umehira, M., High Performance Coherent Demodulator for Wireless ATM Systemsoffset-QPSK simultaneous carrier and bit-timing recovery scheme[C], Vehicular Technology Conference, 1997 IEEE 47th, Vol. 1, pp. 290-294.

[15] K. Tsubono, 300-m laser interferometer gravitational wave detector (TAMA300) in Japan, in Gravitational Wave Experiments, proceedings of the Edoardo Amaldi Conference, World Scientific, pp. 112-114, 1994.

[16] B. Barish and R. Weiss, LIGO and the detection of gravitational waves, Phys. Today, 52, N10, pp. 44-50, October 1999.

[17] P. F. Michelson, Mon. Not. Roy. Astron. Soc. 227, 933 (1987).

[18] N. Christensen, Measuring the stochastic gravitational radiation background with laser interferometric antennas, Phys. Rev. D46, pp. 5250-5266, 1992.

[19] E.E. Flanagan, The Sensitivity of the laser interferometer gravitational wave observatory (LIGO) to a stochastic background, and its dependence on the detector orientations, Phys. Rev. D48, pp. 2389 2407, 1993.

[20] B. Allen, The stochastic gravity-wave background: sources and detection, in Proceedings of the Les Houches School on Astrophysical Sources of Gravitational Waves, eds. J.A. Marck and J.P. Lasota, Cambridge, pp. 373-417, 1995.

\section{AUTHOR}

Senior Manager, Access Network Technology Department, Korea Telecom, Seoul, Korea

(C) 2021 By AIRCC Publishing Corporation. This article is published under the Creative Commons Attribution (CC BY) license. 